\title{
Adult Obesity and the Burden of Disability throughout Life
}

\author{
Anna Peeters, Luc Bonneux, Wilma J. Nusselder, Chris De Laet, and Jan J. Barendregt
}

\begin{abstract}
PEETERS, ANNA, LUC BONNEUX, WILMA J. NUSSELDER, CHRIS DE LAET, AND JAN J. BARENDREGT. Adult obesity and the burden of disability throughout life. Obes Res. 2004;12:1145-1151.

Objective: To analyze the prevalence of disability throughout life and life expectancy free of disability, associated with obesity at ages 30 to 49 years.
\end{abstract}

Research Methods and Procedures: We used 46 and 20 years of mortality follow-up, respectively, for 3521 Original and 3013 Offspring Framingham Heart Study participants 30 to 49 years and classified as normal weight, overweight, or obese at baseline. Disability measures were available between 36 and 46 years of follow-up for 1352 Original participants and at 20 years of follow-up for 2268 Offspring participants. We measured the odds of disability in the Original cohort after 46 years follow-up, and we estimated life expectancy with and without disability from age 50 . Two disability measures were used, one representing limitations with mobility only and the second representing limitations with activities of daily living (ADL).

Results: Obesity at ages 30 to 49 years was associated with a 2.01-fold increase in the odds of ADL limitations 46 years later. Nonsmoking adults who were obese between 30 and 49 years lived 5.70 (95\% confidence interval, 4.11 to 7.35 ) (men) and 5.02 (95\% confidence interval, 3.36 to 6.61 ) (women) fewer years free of ADL limitations from age 50 than their normal-weight counterparts. There was no significant difference in the total number of years lived with disability throughout life between those obese or normal

Received for review August 5, 2003.

Accepted in final form May 17, 2004

The costs of publication of this article were defrayed, in part, by the payment of page charges. This article must, therefore, be hereby marked "advertisement" in accordance with 18 U.S.C. Section 1734 solely to indicate this fact.

Department of Public Health, Erasmus MC, Rotterdam, The Netherlands.

Address correspondence to Anna Peeters, Department of Epidemiology and Preventive Medicine, Monash University, Central and Eastern Clinical School, Alfred Hospital, Commercial Road, Melbourne, Victoria 3004, Australia.

E-mail: anna.peeters@med.monash.edu.au

Copyright () 2004 NAASO weight, due to both higher disability prevalence and higher mortality in the obese population.

Discussion: Obesity in adulthood is associated with an increased risk of disability throughout life and a reduction in the length of time spent free of disability, but no substantial change in the length of time spent with disability.

Key words: functional disability, aging, life expectancy, adult overweight

\section{Introduction}

The prevalence of obesity is increasing rapidly in young adults (1-3). With the uncertainties surrounding weight loss strategies and associated health benefits, prevention before adulthood has the greatest chance of reducing the health burden of obesity (3-5). To understand the potential impact of public health and lifestyle choices at these young ages, an awareness of the long-term health consequences of obesity is needed. We have recently described that obesity at 30 to 49 years is associated with an $\sim 6$-year lesser life expectancy when compared with that of normal weight individuals (6). However, equally, if not more, powerful factors in making lifestyle choices are disability and functional dependence at older ages. These are particularly important for obesity, where increased mortality is only one part of its disease burden (7).

Obesity has been suggested to lead to an increased risk of disability through a range of mechanisms, including skeletal stress and atherogenesis (8). Because obesity in adulthood predicts a range of risk factors (obesity and hypertension) and diseases (diabetes, cardiovascular disease, and osteoarthritis) at older ages that are associated with increased disability (9-11), we expect that adult obesity is associated with greater disability at older ages. At least three studies have shown that obesity in those 25 to 74 years can predict increased disability up to 20 years later $(8,9,12)$. However, the wide age ranges at baseline and the medium-term follow-up periods in these studies do not allow us to determine whether obesity before middle age continues to predict increased disability in old age. The impact of the potentially 
increased age-specific risk of disability on the lifetime experience of disability is also unknown. Because obesity is associated with a large decrease in life expectancy, and because the risk of disability increases sharply with age, those obese before middle age may actually live fewer years disabled than the normal-weight population.

Using long-term follow-up from the Framingham Heart Studies (FHSs), ${ }^{1}$ we aimed to determine to what extent overweight and obesity in adulthood (30 to 49 years) are associated with an increased risk of disability throughout life. We also aimed to determine whether overweight and obesity before middle age are associated with a difference in the duration of life spent with disability compared with those who are normal weight.

\section{Research Methods and Procedures}

\section{Data}

The Original FHS has followed 5209 adults, ages 28 through 62 years, since 1948 (13). We used data from the first 24 biennial exams (46 years follow-up). To capture disability at younger ages, we used the Offspring study, which used similar methods to follow 5124 individuals from $\sim 1970$. We used 20-year follow-up data. We selected persons 30 to 49 years of age [ $n=3607$ (Original), $n=3058$ (Offspring)] with complete baseline measures for height, weight, and smoking status and excluded those underweight $\left(\right.$ BMI $\left.<18.5 \mathrm{~kg} / \mathrm{m}^{2}\right)$ at baseline $(13,14)$. This resulted in 3521 Offspring and 3013 Original participants eligible at baseline. Mortality data were available throughout follow-up for all participants. BMI was calculated as weight in kilograms/height in meters squared. We defined three BMI categories: normal weight, BMI of 18.5 to $24.9 \mathrm{~kg} / \mathrm{m}^{2}$; overweight, BMI of 25 to $29.9 \mathrm{~kg} / \mathrm{m}^{2}$; and obese, BMI $\geq 30$ $\mathrm{kg} / \mathrm{m}^{2}$.

Consistent data on disability were not collected in the Original study until exam 19 (36 years postbaseline) and in the Offspring study until exam 5 (20 years postbaseline), by which time $43 \%$ of the Original cohort and $9 \%$ of the Offspring cohort had died. Figure 1 illustrates the time scheme of the data used in this study. The analyses on disability involved those 1352 Original and 2268 Offspring participants who had complete responses for the disability measures at exams 19 and 5, respectively.

We analyzed the effect of potential confounders on the relationship between obesity and disability (15-17) using complete cases. Baseline measures of mean systolic and mean diastolic blood pressure, history of cardiovascular disease, and diabetes status were available for all participants. Level of education was available for 1318 (97\%) Original participants and 1970 (87\%) Offspring partici-

\footnotetext{
${ }^{1}$ Nonstandard abbreviations: FHS, Framingham Heart Study; ADL, activities of daily living; LE, life expectancy; CI, confidence interval.
}
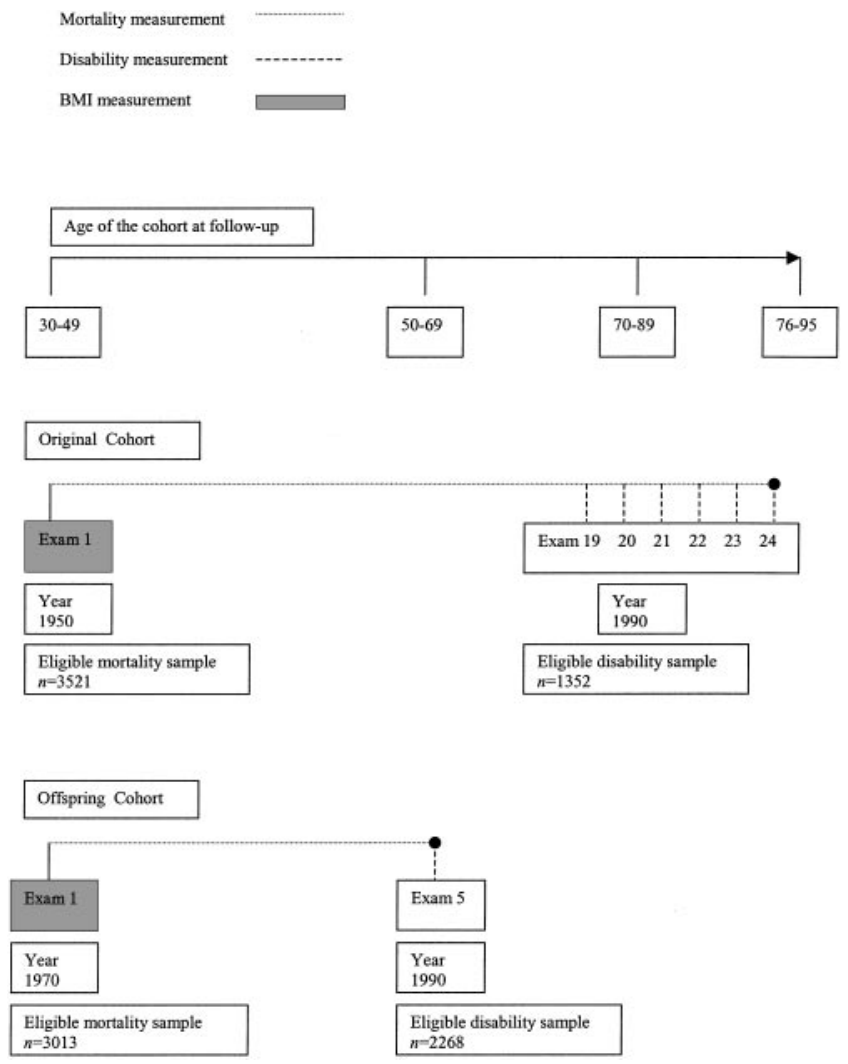

Figure 1: Schematic representation of the data collected from the FHS Original and Offspring cohorts. Body mass index was defined at baseline (exam 1) for both studies. Mortality information was collected throughout the entire follow-up for each study. Disability information was collected at each of the illustrated exams after exam 1 (exams 19 to 24 Original and exam 5 Offspring).

pants. Smoking status was defined as self-reported current smoker, ex-smoker, or never smoker and was recorded at 99.7\% (Original) and 99.9\% (Offspring) of exams where disability status was measured.

\section{Disability}

Disability was measured consistently at 36 (exam 19), 38 (exam 20), 40 (exam 21), 42 (exam 22), 44 (exam 23), and 46 (exam 24) years postbaseline in the Original study and at 20 years postbaseline (exam 5) in the Offspring study. Disability scores were derived from the answers to the question (18-20), "In a normal day, how do you carry out the following activities?" for each of 6 activities. The responses were: 0 , with no help; 1 , using a device; 2 , with human assistance; and 3, dependent. The activities were: a, dressing; b, grooming/bathing; c, feeding/eating; d, transferring (defined as getting in and out of a chair); e, walking on a level surface (50 yards); and f, walking up and down one flight of stairs (five steps). In exam 19, question $\mathrm{f}$ was 10 steps and thereafter 5. Question c was worded as feeding 
(defined as pouring and drinking a glass of water) in exams 19 and 20, eating in exam 21, eating food and drinking liquids in exams 22 to 24 , and eating in the Offspring study.

The prevalence of dichotomized disability scores was analyzed. For dichotomization, disability was defined as nonlimited (response 0) vs. limited (responses 1 to 3 ). At a given age, respondents were defined as mobility only-limited if they had a minimum of one of the two questions e and $\mathrm{f}$ categorized as limited and as activities of daily living (ADL)-limited if they had a minimum of one of the four questions a to $\mathrm{d}$ categorized as limited. The majority of those categorized as ADL-limited also had limitations in walking on a level surface or walking up and down a flight of stairs. To analyze the level of age-associated disability at all ages from 50 years, we combined the data from all of the relevant follow-up exams: the six Original exams between 36 and 46 years follow-up and the single Offspring exam at 20 years follow-up.

\section{Measures of Association}

Odds ratios for disability by baseline BMI status were estimated using logistic regression (SPSS 10, SPSS Inc. Chicago, IL, USA) on the dichotomized disability measures. They were estimated after 46 years of follow-up of the Original study, adjusting for age (including age and age squared) and sex. Potential confounding by smoking status (both baseline and at the exam of disability measurement) and education was addressed by entering these variables into the analyses. Potential confounding by hypertension (systolic blood pressure $\geq 140 \mathrm{mmHg}$ or diastolic blood pressure $\geq 90 \mathrm{mmHg}$ ), diabetes, and cardiovascular disease at baseline was addressed by selecting the population free of all these conditions at baseline and repeating the analyses.

\section{Life Expectancy with and without Disability}

Life tables from age 50 were estimated from the combined Original and Offspring cases eligible at baseline $(n=$ 6534 for mortality follow-up and 3620 for disability prevalence) (Figure 1). The life tables were based on the agespecific mortality rates and disability prevalence of this combined cohort. Because approaching mortality may artificially increase the mortality risks of belonging to the normal weight group relative to the overweight or obese group, we selected cases surviving at least two years after baseline. Because smoking potentially modifies the effect of BMI on mortality $(6,21,22)$, we estimated our life tables separately on baseline nonsmokers $(n=2917)$ and smokers $(n=3589)$. Life table estimation was done in S-plus 2000 (MathSoft Inc., WA, USA). Life tables were derived from the estimated age-specific mortality rates of each weight cohort (6). Poisson regression was used to estimate agespecific mortality rates between ages 50 and 90 based on sex, age at follow-up, and BMI category (see supplemental data available on Obesity Research web site for general fit).
The analyses included a sex-by-age interaction term and assumed an exponential relationship between the mortality rate and age $(23,24)$. As neither age-by-BMI nor sex-byBMI interaction terms were significant, we did not stratify by sex or BMI group. Life expectancy at age 90 was assumed to be that of Massachusetts men or women in 1990 for all BMI categories (25).

Combining all of the available disability measures, logistic regression was used to estimate the age-specific disability prevalence between 50 and 90 years for each weight cohort. Sex, age, age ${ }^{2}$, sex-by-age interaction terms, and BMI category were used to predict disability prevalence (see supplemental data available on Obesity Research web site for fit). Again, as neither age-by-BMI nor sex-by-BMI interaction terms were significant, we did not stratify by sex or BMI group. The age-specific disability prevalence was combined with life tables derived from the age-specific mortality rates, according to the Sullivan method (26), producing life tables for each sex, smoking status, and BMI group stratum. In essence, the life table follows a cohort of people defined by BMI at ages 30 to 49 years throughout life. At each age, it represents the mortality rate and disability prevalence of this cohort as it ages. The life table output was life expectancy (LE) with and without disability from age 50 or age 70 . Confidence intervals (CIs) for the life table measures were calculated using a 5000 replicate bootstrap procedure. We report the bootstrap bias-correct, adjusted $95 \%$ CI (27).

\section{Results}

Survival to the first exam at which disability was measured was inversely related to BMI category. However, among survivors, the response rate at this exam did not differ substantially by BMI category (Table 1). Among responders, the obese cohort had the lowest levels of education, the lowest proportion of smokers, and the highest levels of blood pressure and prevalent cardiovascular disease and hypertension (Table 1). Both the overweight and obese cohorts had similar mean age, approximately one year older than the normal weight cohorts.

Obesity at ages 30 to 49 years was associated with a 2.21-fold (95\% CI, 0.97 to 5.07) increased odds of mobility only-limitations and a 2.01-fold (95\% CI, 1.01 to 4.03) increased odds of ADL-limitations after 46 years of followup, compared with those of individuals with normal weight, after adjustment for age and sex. Overweight was associated with a 1.30-fold ( $95 \%$ CI, 0.88 to 1.92 ) increased odds of mobility only-limitations and a 1.50-fold (95\% CI, 1.04 to 2.17) increased odds of ADL-limitations after 46 years of follow-up. Similar odds ratios were seen in analyses also adjusting for education and smoking status and in analyses selecting those free of baseline hypertension, diabetes, and 
Table 1. Baseline and follow-up characteristics of those with full disability measures at 36 years (original) or 20 years (offspring) postbaseline

\begin{tabular}{|c|c|c|c|}
\hline $\begin{array}{l}\text { Baseline weight group } \\
\text { (at ages } 30 \text { to } 49 \text { ) }\end{array}$ & Normal weight & Overweight & Obese \\
\hline \multicolumn{4}{|l|}{ Original cohort } \\
\hline$n$ & 796 & 447 & 109 \\
\hline \multicolumn{4}{|l|}{ Baseline } \\
\hline Mean age & 37.07 & 38.20 & 38.17 \\
\hline Mean BMI & 22.42 & 27.01 & 33.18 \\
\hline Male (\%) & 33 & 51 & 42 \\
\hline Current smoker (\%) & 56 & 52 & 39 \\
\hline Not high school graduate (\%) & 28 & 39 & 44 \\
\hline Mean systolic blood pressure & 122.17 & 128.66 & 139.39 \\
\hline History of cardiovascular disease $(\%)$ & 0.38 & 0.67 & 1.83 \\
\hline History of diabetes $(\%)$ & 0.50 & 0.00 & 0.00 \\
\hline \multicolumn{4}{|l|}{ Follow-up 36 years } \\
\hline Response rate at exam $19(\%)^{*}$ & 69 & 68 & 69 \\
\hline Mean BMI & 25.01 & 28.23 & 32.36 \\
\hline \multicolumn{4}{|l|}{ Offspring cohort } \\
\hline$n$ & 1104 & 849 & 315 \\
\hline \multicolumn{4}{|l|}{ Baseline } \\
\hline Age & 38.19 & 39.31 & 39.72 \\
\hline BMI & 22.52 & 27.17 & 33.46 \\
\hline Male $(\%)$ & 27 & 70 & 60 \\
\hline Current smoker (\%) & 43 & 42 & 41 \\
\hline Not high school graduate (\%) & 6 & 8 & 14 \\
\hline Mean systolic blood pressure & 117.37 & 123.58 & 132.23 \\
\hline History of cardiovascular disease (\%) & 0.36 & 1.18 & 1.59 \\
\hline History of diabetes $(\%)$ & 0.72 & 0.59 & 3.48 \\
\hline \multicolumn{4}{|l|}{ Follow-up 20 years } \\
\hline Response rate at exam $5(\%)^{*}$ & 84 & 82 & 80 \\
\hline Mean BMI & 24.95 & 28.85 & 34.78 \\
\hline
\end{tabular}

cardiovascular disease (data not shown). Consequently, we used the entire population adjusted only for age and sex in subsequent analyses.

In baseline nonsmokers, the LE of the obese population at age 50 was 5.86 (95\% CI, 4.12 to 7.63 ) (men) and 5.57 (95\% CI, 3.82 to 7.26 ) (women) years fewer than of those with normal weight (Figure 2). The LE of the overweight population at age 50 was 2.53 (95\% CI, 1.07 to 3.76) (men) and 2.33 (95\% CI, 0.92 to 3.45) (women) years fewer than of those with normal weight. The increased risks of mortality and disability were reflected in the overweight and obese populations having significantly lower LE free of disability than the normal weight population. Obese nonsmokers lived 5.70 (95\% CI, 4.11 to 7.35 ) (men) and 5.02
(95\% CI, 3.36 to 6.61) (women) fewer years free of ADLlimitations and 6.02 (95\% CI, 4.35 to 7.61) (men) and 5.53 (95\% CI, 3.76 to 7.34) (women) fewer years free of any (mobility or ADL) limitations from age 50 compared with normal weight nonsmokers. Overweight nonsmokers lived 2.48 (95\% CI, 1.18 to 3.65) (men), and 2.13 (95\% CI, 0.90 to 3.23) (women) fewer years free of ADL-limitations and 2.00 (95\% CI, 0.80 to 3.16) (men), and 1.54 (95\% CI, 0.35 to 2.65) (women) fewer years free of any (mobility or ADL) limitations from age 50 (Figure 2). However, due to the higher mortality in the obese and overweight groups, there was no significant difference in the years lived with disability (mobility or ADL limitations) between those overweight or obese and those normal weight at baseline. 


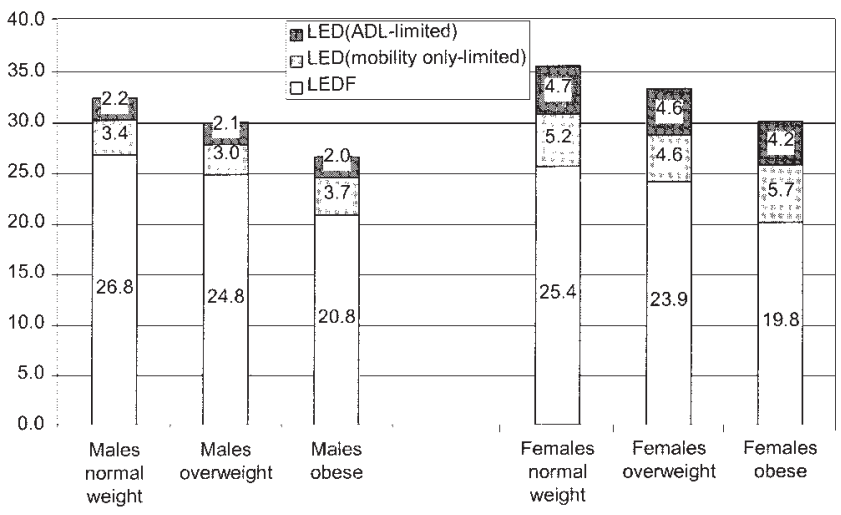

Figure 2: Expected years of life lived with (LED) and without (LEDF) disability from age 50 according to sex and BMI category (all nonsmokers at baseline). The life expectancy with disability is divided into limitations in mobility only and limitations in ADL.

The nonsignificant differences in LE with disability are a balance of more years at younger ages and fewer years at older ages for the overweight and obese populations (Figure 3 ). From age 70, those obese or overweight at ages 30 to 49 years lived fewer years with disability than the normal weight population, because of their higher mortality. Where the point estimates for the life expectancy with disability tended to differ slightly between weight groups, we cannot distinguish whether they represent true differences, nonsignificant due to low power, or a lack of a true difference.

For smokers the general changes in LE were the same as for nonsmokers, with significant losses of LE free of disability, but slight nonsignificant changes in LE with disability associated with overweight and obesity (data not shown).

\section{Discussion}

Obesity at ages 30 to 49 years was associated with an $\sim 2$-fold increase in the odds of disability (significant for ADL-limitations and nonsignificant for mobility only-limitations) 46 years later. Overweight was associated with a significantly increased odds of ADL-limitations, but not mobility-only limitations. There was no change in these odds of disability after taking a population free of hypertension, cardiovascular disease, and diabetes at baseline, suggesting that any causal pathway involving these factors begins with obesity. The life course result of these increased risks is that the 6-year difference in LE from age 50 between obese and normal weight nonsmoking adults is entirely a loss of years free of disability.

The increased risk of disability associated with obesity described here is comparable to a number of cross-sectional and shorter-term follow-up studies (28-30). Other studies have also found only moderate associations of overweight with disability risk $(8,28-30)$. The work presented here

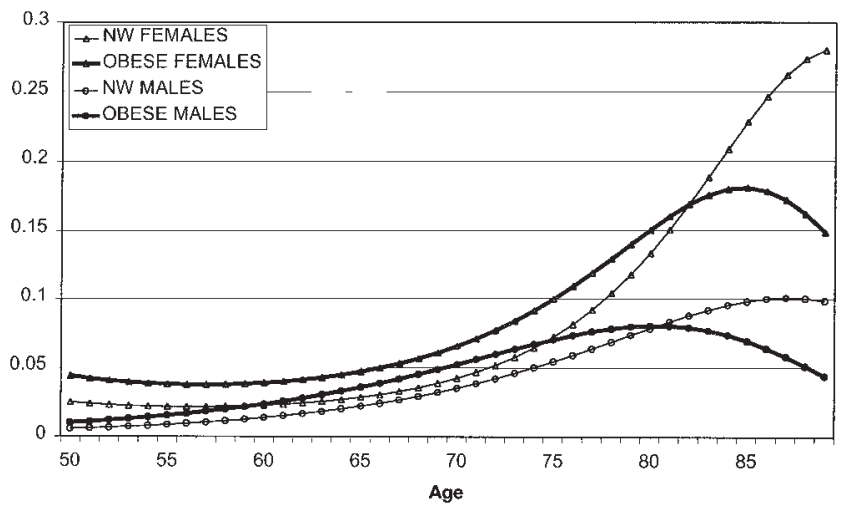

Figure 3: Life years lived with limitations in ADL (dressing, bathing, eating, or transfer) at each year of age by sex and BMI category (in nonsmokers). NW, normal weight.

demonstrated that these previously observed risks can also be predicted for old age by obesity before middle age.

Empirically translating cohort measures of risk into LE with and without disability requires long-term follow-up data (31). This has the necessary limitation that the population is not current. The Original cohort was followed from 1948, so improved prevention and treatment at younger ages may now lead to less mortality and disability associated with obesity. However, through combining the Offspring and Original cohorts, the results presented here were based primarily on mortality and disability data collected from 1970 onwards. It is also known that the Original cohort was a healthy, selected cohort, which may approximate recent populations more closely $(22,32)$.

Another limitation of this study is the combination of the two cohorts. This was done to enable inclusion of disability at ages younger than 70 , as we expect any extra disability experienced by the obese population to occur at younger ages. These cohorts have been previously combined for cardiovascular risk prediction (33), and as related populations, they have many similar characteristics. Our assumption is that the age-specific mortality and disability rates estimated from these 2 cohorts represent those of cohorts who are normal weight, overweight, or obese at ages 30 to 49 years. Although the two cohorts were followed from different periods, the estimated disability prevalence and mortality rates generally represented both cohorts well (see additional figures as supplemental data available on Obesity Research web site). One exception was that in the few cases where there was an overlap of ages between the two cohorts, we found a higher disability prevalence in the Offspring cohort. In contrast to these findings, a previous study found that the Original cohort had more disability than the Offspring cohort (34). While we used identical data to that study for the Offspring cohort, we did not have the data used for the Original cohort, which was measured 20 years earlier than our first Original disability data. The reason for the 
different disability prevalences between the two cohorts in our study at ages 70 to 75 years remains unclear and may be due simply to chance as the numbers involved at these ages are low.

Finally, there are a number of data limitations that need consideration. First, the Framingham cohorts consist of white Americans, so these results are not generalizable to other ethnic populations. Second, self-reported disability measures were used. As there is no evidence to date of differential accuracy by BMI category in reporting of disability, random misclassification may have diluted some of the true differences in disability, but the effect of such random misclassification would not be expected to change our conclusions. Third, while we adjusted for baseline smoking and education status, we were unable to adjust for other potential confounders, such as physical activity, which have an effect on disability and mortality independently of obesity. While the high correlation between physical inactivity and obesity means that our results are probably an acceptable description of the association between adult obesity and lifetime disability, they do not tell us the independent effect of the excess weight alone. Finally, low power meant that we were unable to reach firm conclusions regarding some possible differences in LE with disability, particularly for the overweight population.

Our results confirm other analyses implying that the increasing prevalence of obesity will lead to higher demands for health care interventions at younger to middle ages (35). In the obese populations, there was twice as much time spent disabled at age 60 as in the normal weight populations. However, this is balanced by increasing demand for disability care at older age in normal weight populations; the obese population dies earlier and so accrues less disability at older ages. Consequently, prevention of obesity should not be expected to reduce population levels of disability.

To decrease the inequalities in LE free of disability, the risks of both mortality and disability throughout life associated with obesity in adulthood need to be reduced. A number of mechanisms probably increase these risks, such as higher future BMI, onset of disease, increased duration of excess weight, decreased possibilities for protective behavior, such as physical activity, and increased tendencies for potentially risky behavior, such as rapid or continual changes in weight. In the sample studied here, the obese population differed from the normal weight population at the time of disability measurement in the expected ways: with a higher BMI and a higher prevalence of diabetes and cardiovascular disease (data not shown). At the same time, as we try to identify effective prevention and "cure" strategies for obesity, we also need to identify effective targets for increasing the life expectancy free of disability in those who remain obese before middle age. Analyzing this pop- ulation for the contribution of future risk profiles to future disability and mortality risks will enable identification of such targets.

Obesity before middle age is associated with an increased risk of future disability and mortality and a sizable decrease in disability-free LE. In terms of LE with disability, the disability and mortality risks balance each other: obesity disables at an earlier age but also kills at an earlier age. Consequently, obesity is not associated with an increase in LE with disability, and we should not expect successful prevention to reduce population disability, particularly at older ages. These results once again underscore the importance of preventing obesity before middle age. But one further priority, given current levels of obesity, should be to decrease the inequalities in healthy life by increasing disability-free LE in those with obesity in adulthood.

\section{Acknowledgments}

In addition to the authors, the Netherlands Epidemiology and Demography Compression of Morbidity Research Group consists of F. Janssen, A. Kunst, J.P. Mackenbach, A.A. Mamun and F.J. Willekens. The authors would like to acknowledge the Framingham Heart Study (FHS) co-ordinators for access to the Original and Offspring Datasets. The FHS is conducted and supported by the National Heart, Lung, and Blood Institute (NHLBI) in collaboration with the FHS Investigators. This study was supported by grants from the Netherlands Heart Foundation and the Netherlands Organization for Scientific Research.

\section{References}

1. Flegal KM, Carroll MD, Ogden CL, Johnson CL. Prevalence and trends in obesity among US adults, 1999-2000. JAMA. 2002;288:1723-7.

2. Flegal KM, Carroll MD, Kuczmarski RJ, Johnson CL. Overweight and obesity in the United States: prevalence and trends, 1960-1994. Int J Obes Relat Metab Disord. 1998;22: $39-47$.

3. World Health Organization. Obesity. Preventing and managing the global epidemic. Report of a WHO Consultation on Obesity, June 3-5, 1997. World Health Organization, Geneva, Switzerland, 1998.

4. National Health and Medical Research Council. Acting on Australia's weight: a strategic plan for the prevention of overweight and obesity. Canberra: Australian Government Publishing Service; 1997.

5. U.S. Department of Health and Human Services. The Surgeon General's call to action to prevent and decrease overweight and obesity. Rockville MD: U.S. Department of Health and Human Services, Public Health Service, Office of the Surgeon General; 2001.

6. Peeters A, Barendregt JJ, Willekens F, Mackenbach JP, Mamun AA, Bonneux L. Obesity in adulthood and its consequences for life expectancy: a life-table analysis. Ann Intern Med. 2003;138:24-32.

7. Manson JE, Bassuk SS. Obesity in the United States: a fresh look at its high toll. JAMA. 2003;289:229-30. 
8. Ferraro KF, Su YP, Gretebeck RJ, Black DR, Badylak SF. Body mass index and disability in adulthood: a 20-year panel study. Am J Public Health. 2002;92:834-40.

9. Pinsky J, Leaverton P, Stokes J. Predictors of good function: the Framingham Study. Journal of Chronic Disease 1987; 40(Supplement 1):159-67S.

10. Hubert HB, Bloch DA, Fries JF. Risk factors for physical disability in an aging cohort: the NHANES I Epidemiologic Followup Study. J Rheumatol. 1993;20:480-8.

11. Heo M, Allison DB, Faith MS, Zhu S, Fontaine KR. Obesity and quality of life: mediating effects of pain and comorbidities. Obes Res. 2003;11:209-16.

12. Guralnik JM, Kaplan GA. Predictors of healthy aging: prospective evidence from the Alameda County study. Am J Public Health. 1989;79:703-8.

13. Dawber T, Meadors G, Moore F. Epidemiological approaches to heart disease: the Framingham Study. Am J Public Health. 1951;41:279-86.

14. Hubert H, Feinleib M, McNamara P, Castelli W. Obesity as an independent risk factor for cardiovascular disease: a $26-$ year follow-up of participants in the Framingham Heart Study. Circulation. 1983;67:968-77.

15. Harris T, Cook EF, Garrison R, Higgins M, Kannel W, Goldman L. Body mass index and mortality among nonsmoking older persons. The Framingham Heart Study. JAMA. 1988; 259:1520-4.

16. Manson JE, Willett WC, Stampfer MJ, et al. Body weight and mortality among women. N Engl J Med. 1995;333:67785.

17. Sobal J, Stunkard AJ. Socioeconomic status and obesity: a review of the literature. Psychol Bull. 1989;105:260-75.

18. Pinsky JL, Branch LG, Jette AM, et al. Framingham Disability Study: relationship of disability to cardiovascular risk factors among persons free of diagnosed cardiovascular disease. Am J Epidemiol. 1985;122:644-56.

19. Katz S, Downs TD, Cash HR, Grotz RC. Progress in development of the index of ADL. Gerontologist. 1970;10:20-30.

20. Branch LG, Katz S, Kniepmann K, Papsidero JA. A prospective study of functional status among community elders. Am J Public Health. 1984;74:266-8.

21. Calle EE, Thun MJ, Petrelli JM, Rodriguez C, Heath CW, Jr. Body-mass index and mortality in a prospective cohort of U.S. adults. N Engl J Med. 1999;341:1097-105.
22. Peeters A, Mamun AA, Willekens F, Bonneux L. A cardiovascular life history. Eur Heart J. 2002;23:458-66.

23. Gompertz B. On the nature of the function expressive of the law of human mortality, and on a new method of determining the value of life contingencies. Philos Trans $R$ Soc 1825;115: 513-85.

24. Kesteloot H, Huang $\mathbf{X}$. On the relationship between human all-cause mortality and age. Eur J Epidemiol 2003; in press.

25. Centers for Disease Control and Prevention. U.S. Decennial Life Tables 1989-91. Volume II, State life tables number 22, Massachusetts.: Department of Health and Human Services, USA; 1998.

26. Sullivan DF. A single index of mortality and morbidity. HSMHA Health Rep. 1971;86:347-54.

27. Efron B, Tibshirani R. An Introduction to the Bootstrap. New York: Chapman \& Hall; 1993.

28. Visscher T. The public health impact of obesity. $\mathrm{PhD}$ thesis. Wageningen: Wageningen University; 2001.

29. Launer LJ, Harris T, Rumpel C, Madans J. Body mass index, weight change, and risk of mobility disability in middle-aged and older women. The epidemiologic follow-up study of NHANES I. JAMA. 1994;271:1093-8.

30. Himes CL. Obesity, disease, and functional limitation in later life. Demography. 2000;37:73-82.

31. Peeters A, Bonneux L, Barendregt J, Nusselder W. Methods of estimating years of life lost due to obesity. JAMA. 2003;289:2941.

32. Cupples L, D'Agostino RB, Kiely D. The Framingham Study. An Epidemiological Investigation of Cardiovascular Disease, Section 34. Some Risk Factors Related to the Annual Incidence of Cardiovascular Disease and Death Using Pooled Repeated Biennial Measurements. Framingham Heart Study, 30 Year Follow-up. Bethesda, MD: National Heart, Lung, and Blood Institute; 1987.

33. Anderson KM, Odell PM, Wilson PW, Kannel WB. Cardiovascular disease risk profiles. Am Heart J. 1991;121(1 Pt 2):293-8.

34. Allaire SH, LaValley MP, Evans SR, et al. Evidence for decline in disability and improved health among persons aged 55 to 70 years: the Framingham Heart Study. Am J Public Health. 1999;89:1678-83.

35. Allison DB, Zannolli R, Narayan KM. The direct health care costs of obesity in the United States. Am J Public Health. 1999;89:1194-9. 